Portland State University

PDXScholar

$12-2020$

\title{
Robust Maximum Coverage Facility Location Problem with Drones Considering Uncertainties in Battery Availability and Consumption
}

Darshan Chauhan

Portland State University, drc9@pdx.edu

Avinash Unnikrishnan

Portland State University, uavinash@pdx.edu

Miguel Figliozzi

Portland State University, figliozzi@pdx.edu

Stephen D. Boyles

The University of Texas at Austin, sboyles@austin.utexas.edu

Follow this and additional works at: https://pdxscholar.library.pdx.edu/cengin_fac

Part of the Civil and Environmental Engineering Commons

Let us know how access to this document benefits you.

\author{
Citation Details \\ Published as: Chauhan DR, Unnikrishnan A, Figliozzi M, Boyles SD. Robust Maximum Coverage Facility \\ Location Problem with Drones Considering Uncertainties in Battery Availability and Consumption. \\ Transportation Research Record. December 2020
}

This Pre-Print is brought to you for free and open access. It has been accepted for inclusion in Civil and Environmental Engineering Faculty Publications and Presentations by an authorized administrator of PDXScholar. Please contact us if we can make this document more accessible: pdxscholar@pdx.edu. 


\section{Robust Maximum Coverage Facility Location Problem with Drones Considering \\ Uncertainties in Battery Availability and Consumption}

3

34 Submission Date: December 7, 2020

\section{Darshan R. Chauhan} University, Portland, OR 97201

Email: drc9@pdx.edu (Corresponding Author)

\section{Avinash Unnikrishnan} University, Portland, OR 97201

Email: uavinash@pdx.edu

Miguel Figliozzi Portland, OR 97201

Email: figliozzi@pdx.edu

\section{Stephen D. Boyles}

Email: sboyles@mail.utexas.edu

Graduate Research Assistant, Department of Civil and Environmental Engineering, Portland State

Associate Professor, Department of Civil and Environmental Engineering, Portland State

Professor, Department of Civil and Environmental Engineering,Portland State University,

Associate Professor, Department of Civil, Architectural, and Environmental Engineering, The University of Texas at Austin, Austin, TX 78712

Word Count: 7589 words +4 table $(s) \times 250=8589$ words 


\section{ABSTRACT}

2 Given a set of spatially distributed demand for a specific commodity, potential facility 3 locations, and drones, an agency is tasked with locating a prespecified number of facilities and as4 signing drones to them to serve the demand while respecting drone range constraints. The agency 5 seeks to maximize the demand served while considering uncertainties in initial battery availability 6 and battery consumption. The facilities have a limited supply of the commodity being distributed 7 and also act as a launching site for drones. Drones undertake one-to-one trips (from located facility 8 to demand location and back) until their available battery energy is exhausted. This paper extends

9 the work done by Chauhan et al. (1) and presents an integer linear programming formulation to 10 maximize coverage using robust optimization framework. The uncertainty in initial battery avail11 ability and battery consumption is modeled using a penalty-based approach and gamma robustness, 12 respectively. A novel robust three stage heuristic $(\mathrm{R} 3 \mathrm{SH})$ is developed which provides objective 13 values which are within $7 \%$ of the average solution reported by MIP solver with a median reduction 14 in computational time of $97 \%$ on average. A Monte Carlo Simulation based testing is performed 15 to evaluate the value of adding robustness to the deterministic problem. The robust model provides 16 higher and more reliable estimates of actual coverage under uncertainty. The average maximum 17 coverage difference between the robust optimization solution and deterministic solution is $8.1 \%$ 18 across all scenarios.

19

Keywords: Drones, UAV, Robust optimization, Battery uncertainty, Facility location, Coverage objective, Energy, Decomposition heuristic 


\section{INTRODUCTION}

Drones are increasingly being considered for diverse applications such as emergency response and disaster management (2-4), agriculture (5-7), and commercial package deliveries (8). Applications of drones and unmanned aerial vehicles (UAVs) are expected to increase in the next few decades as they can access locations with limited or damaged roadway infrastructure. Moreover, technological advances in lighter frames (9), control algorithms (10), batteries (11), and removal of existing flight regulations (12) is expected to expedite large scale drone and UAV adoption.

Drones have limited range restricted by battery capacity. Often the maximum range decreases with the payload. Therefore, drone launching facilities are required to make deliveries in a large scale urban area. Recently, Chauhan et al. (1) developed a mixed-integer linear program called Maximum Coverage Facility Location Problem with Drones (MCFLPD) and an efficient three-stage heuristic (3SH) to select a pre-specified number of drone launching sites with resource capacities, allocation of pre-specified number of drones to each launching site to make deliveries, and assignment of spatially distributed demand locations to each launching site and drones. The MCFLPD formulation assumed a deterministic battery capacity and consumption rate. It is well known that the battery capacity and consumption can vary significantly based on weather - temperature and wind conditions, which can constrain the maximum range. This paper extends the MCFLPD problem by using a robust optimization framework to capture the uncertainty in battery capacity and consumption. This paper develops a new Robust Maximum Coverage Facility Location Problem with Drones (RMCFLPD) to capture uncertainty in battery capacity and consumption rate. An efficient decomposition solution procedure called Robust Three-stage Heuristic (R3SH) is provided. Computational analysis is performed on a real-world case study in Portland, OR which demonstrates the value of the heuristic and the need for capturing battery capacity and consumption rate uncertainty. The RMCFLPD model studied in this work is the first to incorporate battery consumption and capacity uncertainty in a framework which models drone energy consumption with payload and distance in a maximum coverage location problem setting. We also provide an efficient decomposition-based solution heuristic, which exploits the problem structure.

The literature review is described next followed by the problem formulation and solution algorithm. Discussion of computational analysis conducted on a real-world case study in Portland, OR is presented next followed by the conclusions and directions for future research.

\section{LITERATURE REVIEW}

Several researchers have focused on developing interesting variants of the traveling salesman and vehicle routing problems focusing on drone applications such as the flying sidekick traveling salesman problem where a drone and truck make deliveries together (13-18). Wang et al. (19), Poikonen et al. (20), Daknama and Kraus (21), and Dayarian et al. (22) study the vehicle routing problem with drones variant. In general, incorporating drones into the existing fleet was found to increase reliability and efficiency. In this paper, we focus on drone-based deliveries only and do not consider the integration of drones into a trucking fleet. Dorling et al. (23) and Choi and Schonfeld (24) study the impact of battery consumption and payload weight on single depot dronebased delivery systems which considers multiple deliveries made by a drone in a single route from a depot. In contrast, the model developed in this work only considers multiple one-to-one deliveries from a pre-specified depot. However, we do model multiple depots. 
Chowdhury et al. (25), Golabi et al. (26), Pulver and Wei (27), and Kim et al. (28) study

2 facility location problems for drone delivery systems in the context of humanitarian logistics and

3 medical supply delivery systems. The RMCFLPD model and solution algorithm distinguish from

4 the works mentioned above in several aspects. Chowdhury et al. (25) consider both trucks and

5 drones, whereas we focus on a pure drone-based delivery system. Pulver and Wei (27) do not

6 model capacity constraints at facilities, energy consumption with payload, and assume one trip

7 per drones, whereas the RMCFLPD model considers all of these aspects. Pulver and Wei (27)

8 and Kim et al. (28) use optimization solvers which may not scale up well to larger instances,

9 whereas this research provides a customized, efficient heuristic. None of the works mentioned

10 above consider the allocation of drones to facilities. This research is an extension of the model

11 and solution algorithm proposed by Chauhan et al. (1) by using a robust optimization paradigm to

12 model battery consumption and battery capacity uncertainty.

Kim et al. (29) use a robust optimization approach to study the impact of air temperature on uncertainty in maximum flight duration. However, Kim et al. (29) do not model variation in energy consumption with payload, allocation of drones to facilities, facility capacity, and use CPLEX to solve the problem. Kim et al. (30) develop a chance constraint formulation using an exponential distribution to model the impact of battery uncertainty on coverage of a location. Unlike Kim et al. (30), the RMCFLPD adopts a robust optimization approach where the battery consumption and capacity is assumed to vary in a pre-specified range and therefore is distribution-free.

Goodchild and Toy (31) and Figliozzi (32) evaluate relative efficiency, energy consumption, and emissions from UAVs relative to trucks. A detailed review of optimization approaches in drone-based delivery systems and applications is provided by (33). Summarizing, this research presents a new robust optimization approach as well as a new efficient heuristic to tackle the facility location problem with drones with battery consumption and capacity uncertainty.

\section{PROBLEM DESCRIPTION}

This section describes a mixed-integer linear programming formulation for the Robust Maximum Coverage Facility Location Problem with Drones (RMCFLPD). Consider a set of locations $I$ each having a demand $d_{i}$ and set of location sites $J$. At the beginning of the planning period, an agency has to pick a maximum of $p$ facilities from the location set $J$ to serve as drone launching sites. At each open facility, resources of mass $U$ are allocated to be distributed to the demand points. The planning agency also has to distribute a set of $K$ drones to the located facilities. We assume that the cost of transporting the drones and resources from a warehouse to each open facility is constant. The drones make one-to-one delivery trips (from the facility location to the demand points and back) until the battery is exhausted. We do not consider one-to-many vehicle routing type trips, which is consistent with the initial applications of drone deliveries by private companies. We also do not consider battery recharging during the planning period and assume that the drone battery is recharged in-between planning periods. The length of the planning period is shorter ( 6 hours to a day or two days) compared to the planning period for a typical facility location problem.

We adopt a robust optimization framework to capture the uncertainty in battery consumption and initial capacity. For each drone $k \in K$, the battery capacity can take any value in the interval $\left[B-f_{k}, B\right]$. To model the robustness in initial battery availability, a penalty of $F_{k}$ is assigned per fractional reduction in the initial battery availability. The conservativeness in battery capacity can be controlled by adjusting the penalty. Higher values of $F_{k}$ lead to more conservative 
1 solutions concerning battery capacity. The battery consumption during one trip between demand

2 point $i \in I$ and facility location $j \in J$ is assumed to be uncertain and can take any value in the inter-

3 val $\left[b_{i j}-\hat{b}_{i j}, b_{i j}+\hat{b}_{i j}\right]$ where $b_{i j}$ is the nominal value and $\hat{b}_{i j}$ is the maximum variation. We adopt

4 the gamma robustness paradigm originally proposed by (34). In the gamma robustness framework,

5 the battery consumption during one trip between demand point $i \in I$ and facility location $j \in J$ can

6 take one of two values - the nominal value $b_{i j}$ or the worst-case value $b_{i j}+\hat{b}_{i j}$. For each drone,

7 we assume that at most $\Gamma_{j k}$ trips are at worst-case battery consumption with the remaining trips at

8 nominal battery consumption. The nomenclature and mathematical programming formulation are

9 presented below.

10 Nomenclature

Sets

11

I Set of all demand points

$J \quad$ Set of all candidate facility locations

$K \quad$ Set of available drones

Indices

12

$i \in I$

$j \in J$

$k \in K$

Parameters

$\eta \quad$ Power transfer efficiency of the drone

$v_{s} \quad$ Lift-to-drag ratio of the drone

$m_{t} \quad$ UAV tare mass, without battery and load

$m_{b} \quad$ UAV battery mass

$d_{i} \quad$ Demand for resource at location $i \in I$ (units same as UAV battery and tare mass)

$c_{i j} \quad$ Distance between demand location $i \in I$ and facility location $j \in J$

13

$b_{i j} \quad$ Nominal battery consumption during one trip between demand point $i \in I$ and facility

$\hat{b}_{i j} \quad$ Variation in battery consumption during one trip between demand point $i$ and facility

$\hat{b}_{i j} \quad$ location $j$

$B \quad$ Maximum usable battery capacity of the drone

$f_{k} \quad$ Maximum decrease in initial battery capacity for drone $k \in K$

$F_{k} \quad$ Penalty associated with decreasing initial battery availability for drone $k \in K$

$p \quad$ Maximum number facilities that can be opened

$U \quad$ Capacity of each located facility (units same as UAV battery and tare mass)

$\Gamma_{j k} \quad$ Maximum number of trips from located facility $j$ by drone $k$ that can achieve worst

r $j k \quad$ case battery consumption

Decision Variables

$x_{i j k} \quad 1$, if demand location $i$ is served by located facility $j$ using drone $k$; and 0 , otherwise

$y_{j} \quad 1$, if candidate facility location $j$ is opened; and 0 , otherwise

$14 \quad z_{j k}$

1 , if located facility $j$ employs drone $k$; and 0 , otherwise

$\gamma_{i j k} \quad 1$, if trip to demand location $i$ from facility location $j$ by drone $k$ assumes worst case battery consumption; and 0 , otherwise

$\delta_{j k} \quad$ fraction of maximum decrease in initial battery capacity $\left(f_{k}\right)$ of drone $k$ employed by facility location $j\left(0 \leq \delta_{j k} \leq 1\right)$ 


\section{Problem Formulation}

$$
\begin{aligned}
\max _{x, y, z, \delta} \sum_{i \in I} \sum_{j \in J} \sum_{k \in K} d_{i} x_{i j k} & +\sum_{j \in J} \sum_{k \in K} F_{k} \delta_{j k} \\
\sum_{j \in J} \sum_{k \in K} x_{i j k} & \leq 1 \quad \forall i \in I \\
\sum_{j \in J} y_{j} & \leq p \\
\left(\max _{\gamma} \sum_{i \in I}\left(b_{i j}+\gamma_{i j k} \hat{b}_{i j}\right) x_{i j k}\right) & \leq\left(B-f_{k} \delta_{j k}\right) z_{j k} \quad \forall j \in J, k \in K \\
\sum_{i \in I} \sum_{k \in K} d_{i} x_{i j k} & \leq U y_{j} \quad \forall j \in J \\
z_{j k} & \leq y_{j} \quad \forall j \in J, k \in K \\
\sum_{j \in J} z_{j k} & \leq 1 \quad \forall k \in K \\
\sum_{i \in I} \gamma_{i j k} & \leq \Gamma_{j k} \quad \forall j \in J, k \in K \\
\delta_{j k} & \leq z_{j k} \quad \forall j \in J, k \in K \\
x_{i j k} & \in\{0,1\} \quad \forall i \in I, j \in J, k \in K \\
y_{j} & \in\{0,1\} \quad \forall j \in J \\
z_{j k} & \in\{0,1\} \quad \forall j \in J, k \in K \\
\gamma_{i j k} & \in\{0,1\} \quad \forall i \in I, j \in J, k \in K \\
\delta_{j k} & \geq 0 \quad \forall j \in J, k \in K
\end{aligned}
$$

The goal of the objective function is to maximize the sum of the demand served by the drones and the penalty for initial battery availability. The penalty term promotes reduction in the initial battery availability, thereby improving robustness. Constraint 2 ensures that a demand point is covered at most once. Constraints 3 and 5 ensure that at most $p$ facilities are opened, and its corresponding capacity constraints are satisfied. Together, constraints 6 and 7 ensure that drones are allocated to open facilities, and each drone is assigned to at most one facility only.

$b_{i j}=\frac{m_{t}+m_{b}+d_{i}}{v_{s} \eta} c_{i j}+\frac{m_{t}+m_{b}}{v_{s} \eta} c_{i j} \quad \forall i \in I, j \in J$

Constraint 4 enforces battery range constraints on all the drones considering battery consumption robustness and reduction in total available battery. Constraint 8 puts a limit on the total number of worst-case battery consumption trips per drone at each facility according to the gamma robustness principle (34). Constraint 9 makes sure that the total battery availability penalty on the drone $k$ located at facility $j$ is only applied if it is placed there. Equations 10-14 are variable definition constraints.

A common assumption in robust optimization is that the uncertainty occurs in such a way that it worsens the decision-maker's objective $(34,35)$, i.e. for a maximization problem, the uncertainty occurs in such a way that it minimizes the objective value. The above formulation can not be solved directly as the maximization in 4 is in direct conflict with overall objective in equation 
1 , a consequence of applying robust optimization. The presence of non-linear terms in equation 4

2 further complicates the problem. To remedy the conflicting objective and non-linear terms $\gamma_{i j k} x_{i j k}$,

3 the optimization problem in equation 4 , with relevant constraints 8 and 13, is dualized. This inner 4 optimization problem in the variable $\gamma\left(S P_{j k}\right)$ is given as:

$$
\begin{gathered}
S P_{j k}=\max _{\gamma} \sum_{i \in I} \hat{b}_{i j} x_{i j k} \gamma_{i j k} \\
\sum_{i \in I} \gamma_{i j k} \leq \Gamma_{j k}
\end{gathered}
$$

$$
\gamma_{i j k} \in\{0,1\} \quad \forall i \in I
$$

5

6

In the above formulation, parameter $\Gamma_{j k}$ is an integer. In case of non-integer values, $\Gamma_{j k}$ can be changed to $\left\lfloor\Gamma_{j k}\right\rfloor$ to retain correctness. The above formulation provides an integer optimal solution when the variable $\gamma$ is linearized. Let, $\theta_{j k}$ and $\mu_{i j k}$ be the dual variables associated with equation 17 and the upper bound of the equation 18 respectively. The dual formulation of the above problem $\left(S P D_{j k}\right)$, can then be written as:

$$
\begin{aligned}
S P D_{j k}=\min _{\mu, \theta}\left(\sum_{i \in I} \mu_{i j k}\right)+\Gamma_{j k} \theta_{j k} & \\
\mu_{i j k}+\theta_{j k} & \geq \hat{b}_{i j} x_{i j k} \quad \forall i \in I \\
\mu_{i j k} & \geq 0 \quad \forall i \in I \\
\theta_{j k} & \geq 0
\end{aligned}
$$

Using strong duality, it can be shown at $S P_{j k}$ and $S P D_{j k}$ have the same optimal value. The product $\delta_{j k} z_{j k}$ (in equation 4) can be simplified and written as only $\boldsymbol{\delta}_{j k}$ because of the presence of constraint 9. Substituting the above changes, the modified RMCFLPD formulation is given as:

$$
\begin{gathered}
\max _{x, y, z, \delta, \mu, \theta} \sum_{i \in I} \sum_{j \in J \in K} \sum_{k \in K} d_{i} x_{i j k}+\sum_{j \in J} \sum_{k \in K} F_{k} \delta_{j k} \\
\sum_{j \in J} \sum_{k \in K} x_{i j k} \leq 1 \quad \forall i \in I \\
\sum_{j \in J} y_{j} \leq p \\
\left(\sum_{i \in I} b_{i j} x_{i j k}\right)+\left(\sum_{i \in I} \mu_{i j k}\right)+\Gamma_{j k} \theta_{j k}+f_{k} \delta_{j k} \leq B z_{j k} \quad \forall j \in J, k \in K \\
\mu_{i j k}+\theta_{j k}-\hat{b}_{i j} x_{i j k} \geq 0 \quad \forall i \in I, j \in J, k \in K \\
\sum_{i \in I} \sum_{k \in K} d_{i} x_{i j k} \leq U y_{j} \quad \forall j \in J \\
z_{j k} \leq y_{j} \quad \forall j \in J, k \in K \\
\sum_{j \in J} z_{j k} \leq 1 \quad \forall k \in K \\
\delta_{j k} \leq z_{j k} \quad \forall j \in J, k \in K \\
x_{i j k} \in\{0,1\} \quad \forall i \in I, j \in J, k \in K \\
y_{j} \in\{0,1\} \quad \forall j \in J
\end{gathered}
$$




$$
\begin{aligned}
z_{j k} & \in\{0,1\} \quad \forall j \in J, k \in K \\
\mu_{i j k} & \geq 0 \quad \forall i \in I, j \in J, k \in K \\
\theta_{j k} & \geq 0 \quad \forall j \in J, k \in K \\
\delta_{j k} & \geq 0 \quad \forall j \in J, k \in K
\end{aligned}
$$

The sum $\left(\sum_{i \in I} \mu_{i j k}+\Gamma_{j k} \theta_{j k}\right)$, in equation 26 , represents the additional battery consumption because of robustness consideration, and the term $f_{k} \delta_{j k}$ is the reduction in the total available battery.

\section{ROBUST THREE STAGE HEURISTIC FOR SOLVING RMCFLPD}

\section{Facility location and demand allocation}

12 In this stage, a facility location problem is solved to determine the facilities to be opened and

The robust three-stage heuristic (R3SH) solves the RMCFLPD in three stages. This method is an extension of the $3 \mathrm{SH}$ heuristic proposed in (1). The first stage is a facility location problem for deciding which facilities to open and matching them with demand points. The second stage is solving the robust knapsack problem, including battery consumption uncertainty and initial battery availability penalty, to allocate drones to open facilities. The final stage is an $r$-exchange heuristic to replace $r$ worst-performing facilities to improve the solution quality. matching demand points to the located facilities. Let, $\bar{J}_{i}$ denote the set of potential facility locations that are within the range of the drone for each demand location $i$, i.e. $\bar{J}_{i}=\left\{j \in J \mid b_{i j} \leq B\right\}$. Also let, $\bar{I}_{j}$ denote the set of demand points $i \in I$ that are within the range of the drone for each facility location $j \in J$, i.e. $\bar{I}_{j}=\left\{i \in I \mid b_{i j} \leq B\right\}$. The sets $\bar{J}_{i}$ and $\bar{I}_{j}$ ensure that the demand points and facilities are within the flying range of each other. The decision variables for the formulation are: (i) $\hat{x}_{i j}$ which takes values 1 if demand point $i \in I$ is assigned to facility $j \in J$ and 0 otherwise, and (ii) $\hat{y}_{j}$ which takes value 1 if facility $j \in J$ is opened and 0 otherwise.

$$
\begin{gathered}
\max _{\hat{x}, \hat{y}} \quad \sum_{i \in I} \sum_{j \in \bar{J}_{i}} \frac{d_{i}}{b_{i j}} \hat{x}_{i j} \\
\sum_{j \in \bar{J}_{i}} \hat{x}_{i j} \leq 1 \quad \forall i \in I \\
\sum_{j \in J} \hat{y}_{j} \leq p \\
\sum_{i \in \bar{I}_{j}} d_{i} \hat{x}_{i j} \leq U y_{j} \quad \forall j \in J \\
\hat{x}_{i j}, \hat{y}_{j} \in\{0,1\} \quad \forall i \in \bar{I}_{j}, j \in J
\end{gathered}
$$

The objective of the formulation, equation 38 , is to maximize the weight of assigned demand points. Constraint 39 makes sure that the demand point is covered by at most one facility. Constraint 40 ensures that no more than $p$ facilities are opened. Constraint 41 enforces the sum of demand assigned to a facility to be less than its capacity.

\section{Repeated application of robust knapsack problems}

Let $\hat{J}$ be the set of facilities opened and $\hat{I}_{j}$ be the set of demand points matched to open facilities, as obtained from the first stage of R3SH. That is, $\hat{J}=\left\{j \in J \mid \hat{y}_{j}=1\right\}$, and $\hat{I}_{j}=\left\{i \in I \mid \hat{x}_{i j}=1\right\}$. In this stage, the drones are allocated to opened facilities to serve demand points by solving a maximum 
1 profit robust knapsack problem. For any facility $j \in \hat{J}$ and drone $k \in K$. the max profit robust 2 knapsack problem is defined as follows:

$$
\begin{gathered}
C_{j}=\max _{x^{\prime}, w^{\prime}}\left(\sum_{i \in \hat{I}_{j}} d_{i} x_{i}^{\prime}\right)+F_{k} w_{j k}^{\prime} \\
\left(\sum_{i \in \hat{I}_{j}} b_{i j} x_{i}^{\prime}\right)+\left(\max _{\left\{i \in S\left|S \subseteq \hat{I}_{j},\right| S \mid \leq \Gamma_{j k}\right\}} \hat{b}_{i j} x_{i}^{\prime}\right)+f_{k} w_{j k}^{\prime} \leq B \\
x_{i}^{\prime} \in\{0,1\} \quad \forall i \in \hat{I}_{j} \\
w_{j k}^{\prime} \in\{0,1\}
\end{gathered}
$$

3 In the above formulation, the variables $x_{i}^{\prime}$ take the value 1 if demand point $i$ is served by 4 drone $k$ from facility $j$ and 0 otherwise. The variable $w_{j k}^{\prime}$ takes the value 1 if the penalty is applied 5 completely to the initial battery availability, and 0 if the penalty is not applied at all. Constraint 644 makes sure that only the demand points satisfying the drone battery constraint are served. $C_{j}$ 7 represents the maximum value of the 0-1 maximum profit robust knapsack problem. The above 8 problem is solved by solving $\left|\hat{I}_{j}\right|-\Gamma_{j k}+1$ ordinary $0-1$ knapsack problems, as shown in Lee et al. 9 (36). Let, $\gamma_{i}^{\prime}$ be 1 if $i \in S$ and 0 if $i \in \hat{I}_{j} \backslash S$. Then, the determination of the non-binary value of the penalty is done in the following manner:

$\delta_{j k}^{\prime}= \begin{cases}1 & ; \text { if } w_{j k}^{\prime}=1 \\ \frac{B-\sum_{i \in \hat{I}_{j}}\left(b_{i j}+\gamma_{i}^{\prime} \hat{b}_{i j}\right) x_{i}^{\prime}}{f_{k}} & ; \text { if } w_{j k}^{\prime}=0\end{cases}$

11 The final objective function value, $C_{j}^{F}$, is then determined as follows:

$C_{j}^{F}= \begin{cases}C_{j} & ; \text { if } \delta_{j k}^{\prime}=1 \\ C_{j}+F_{k} \delta_{j k}^{\prime} & ; \text { if } \delta_{j k}^{\prime}<1\end{cases}$

12

$C_{j}^{F}$ represents the maximum value of the sum of demand satisfaction and the penalty possible from facility $j$ and its corresponding demand locations $\hat{I}_{j}$. The steps involved in R3SH are given as follows:

- The best facility for the allocation of the first drone is determined by solving $|\hat{J}|$ maximum profit robust knapsack problems, once for each $j \in \hat{J}$. Let $j^{\prime}$ be the facility with a maximum value of $C_{j}^{F}$. Allot the first drone to $j^{\prime}$ and remove the demand points served by the first drone from the set $\hat{I}_{j^{\prime}}$. Assign penalty variable $\delta_{j^{\prime} k}^{\prime}$ to the first drone.

- Solve the maximum profit robust knapsack problem for $j^{\prime}$ using the updated $\hat{I}_{j^{\prime}}$, and determine the new value for $C_{j^{\prime}}^{F}$. Now let $j^{\prime \prime}$ be the facility with the maximum value of $C_{j}^{F}$. Allot the second drone to $j^{\prime \prime}$ and remove the demand points served by the second drone from the set $\hat{I}_{j^{\prime \prime}}$. Assign penalty variable $\delta_{j^{\prime \prime} k}^{\prime}$ to the second drone.

- Repeat the above step until all the drones are used or all demand points are satisfied. This would result in a maximum of $|K|-1$ repetitions. If no more demand points can be satisfied, then, assign the remaining drones to the facility with a maximum $C_{j}^{F}$ value and set the corresponding $\delta_{j k}^{\prime}$ values to 1 . 
$1 \quad r$-exchange heuristic

2 In the third stage, a local exchange heuristic is employed to improve solutions. Set $\hat{J_{0}}=\hat{J}$, and 3 determine the sum of demand served and penalty for each open facility. The $r$ facilities with least 4 sum of demand served and penalty are selected to be closed and are removed from $\hat{J}$. $\hat{J}$ is then 5 updated by adding $r$ facilities randomly chosen from the $|J|-p+r$ facilities that are currently 6 closed. Update the sets $\bar{J}_{i}=\left\{j \in \hat{J} \mid b_{i j} \leq B\right\}, \forall i \in I$ and $\bar{I}_{j}=\left\{i \in I \mid b_{i j} \leq B\right\}, \forall j \in \hat{J}$. The 7 demand points are then matched to the open facilities by solving the following problem:

$$
\begin{aligned}
& \max _{\hat{x}} \sum_{i \in I} \sum_{j \in \bar{J}_{i}} \frac{d_{i}}{b_{i j}} \hat{x}_{i j} \\
& \sum_{j \in \bar{J}_{i}} \hat{x}_{i j} \leq 1 \quad \forall i \in I \\
& \sum_{i \in \bar{I}_{j}} d_{i} \hat{x}_{i j} \leq U \hat{y}_{j} \quad \forall j \in \hat{J} \\
& \hat{x}_{i j} \in\{0,1\} \quad \forall i \in \bar{I}_{j}, j \in \hat{J}
\end{aligned}
$$

where $\hat{y}_{j}=1, \forall j \in \hat{J}$ and 0 otherwise, and is not a decision variable in the above formulation. Once the above demand allocation problem is solved, the second stage of solving $|\hat{J}|+|K|-1$ maximum profit robust knapsack problems is repeated. If the sum of total demand served and battery capacity penalty is found to be better than the previous best solution, then $\hat{J}_{0}$ is updated to the current set of open facilities. If there was no improvement, then the previous best solution and the set of open facilities $\hat{J}_{0}$ is adopted, and new $r$ facilities are randomly chosen. This $r$-exchange heuristic is repeated for a prespecified number of times.

Proposition 1: The solution generated at the end of stage 2 of R3SH (i.e., repeated application of robust knapsack problems) is a feasible lower bound of RMCFLPD.

Proof: In stage 2, the variable $\hat{x}_{i j}$ (from stage 1) helps determine $\hat{I}_{j}$, a set of demand points that can be served from facility $j$ only. If a demand point is served by the drone, it is removed from the set $\hat{I}_{j}$. Therefore, the following inequality is valid:

$\sum_{k \in K} x_{i j k} \leq \hat{x}_{i j} \quad \forall i \in I, j \in J$

Now, consider the stage 1 problem (equations 38-42). Using valid inequality 53, if equations 39, 40, and 41 are satisfied in R3SH, the corresponding RMCFLPD equations 24, 25, and 28, respectively, are also satisfied. Additionally, a preliminary precaution is taken that $\hat{x}_{i j}$ can assume the value 1 only if $b_{i j} \leq B$. This ensures that only plausible deliveries are considered. Therefore, stage 1 solution provides with feasible facility locations and demand allocation to facilities.

Stage 2 of R3SH tries to allot a drone to a facility and allocate demand points that would be served by the drone. The maximum profit robust knapsack problem (equations 43-46) considers a drone alloted to facility $j$ and determines the maximum sum of demand and battery availability penalty that can be achieved. Constraint 44 is equivalent to constraint 4 (or the set of constraints 26 and 27 in RMCFLPD). The robust knapsack problem considered here was first introduced in (37). The solution algorithm to solve robust knapsack problem is proposed by (36), who also prove that the algorithm ensures optimality. Therefore, allocation of demand points to a drone located at $j$ is always feasible. Also, it is easy to notice that value of $\delta_{j k}^{\prime}$ found in equation 47 always lies in the range [0,1] such that constraint 4 (or equivalently constraints 26 and 27 in RMCFLPD) always remain valid. As a drone is always allotted only to one of the open facilities represented by the set 
$1 \hat{J}$, equations 30 and 31 in RMCFLPD are also satisfied.

2 As all the constraints in RMCFLPD are satisfied by R3SH at the end of Stage 2, the gener-

3 ated solution is feasible, and therefore, a valid lower bound of RMCFLPD.

Corollary 1: The solution generated at the end of stage 3 of R3SH (i.e. $r$-exchange heuristic) is at least as good as the solution generated in stage 2 .

Proof: The optimization problem in stage 3 of $\mathrm{R} 3 \mathrm{SH}$, is essentially the stage 1 problem with variables $\hat{y}$ fixed. This is followed by repeated application of robust knapsacks, i.e. stage 2 of $\mathrm{R} 3 \mathrm{SH}$. Therefore, following Proposition 1, the solution obtained at the end of stage 3 is feasible. Now, if the solution found at the end of reiterated stage 2 is worse than the previous best, it is discarded, and previous best solution is used again for stage 3 . If the solution is better than the previous best solution, then the previous best is updated. Therefore, the solution obtained at the completion of stage 3 is at least as good as the one obtained at the end of stage 2 .

\section{NUMERICAL ANALYSIS}

Computational analysis on the impact of drone battery consumption and capacity uncertainty on drone-based deliveries for short term planning periods is performed on a case study based in the Portland Metropolitan Area (1). The Portland Metropolitan area spans a total of five counties in the state of Oregon (Clackamas, Columbia, Multnomah, Washington, and Yamhill) and two counties in the state of Washington (Clark, and Skamania). The centroids of the ZIP Code Tabulated Areas (ZCTAs) in these seven counties are considered to be the demand locations for the study. The community centers across the Portland Metro are considered as the potential facility locations, as they provide enough space for storing resources and launching drones. There are 122 demand locations and 104 candidate facility location sites in the case study, and none of them overlap with another. The demand locations and the candidate facility locations are shown in Figure 1. The resource requirement at demand locations varies uniformly between $1 \mathrm{~kg}$ and $5 \mathrm{~kg}$ in discrete intervals of $0.25 \mathrm{~kg}$. The values chosen here are the same as in Chauhan et al. (1), and the total demand is $366.5 \mathrm{~kg}$. The facilities are assumed to operate at an average of $80 \%$ capacity efficiency. The capacities can then be generated as in Pirkul and Schilling (38):

$U=\frac{\sum_{i \in I} d_{i}}{0.8 p}$ where, the numerator denotes the total demand for the resource, and $p$ denotes the maximum number of facilities that can be located. In the case study, $p$ takes values from 5 to 30 in multiples of 5. The distance between the demand locations and candidate facility locations is assumed to be the planar Euclidean distance between them, as drones usually travel in straight lines. Currently, the effect of tall buildings, mountains, "no-drone zones" (12) and other obstacles on drone trajectory is not considered, and it can be a possible future extension. The nominal battery consumption $\left(b_{i j}\right)$ for a trip to demand location $i$ from a facility location $j$ is a function of the distance between them and the demand for the resource at location $i$ and can be calculated using equation 15 . The variation in battery consumption $\left(\hat{b}_{i j}\right)$ is assumed to be strongly and positively correlated to nominal battery consumption $\left(b_{i j}\right)$ and is an integer chosen randomly in the interval $\left[0.1 b_{i j}, 0.3 b_{i j}\right]$ (currently chosen values of $\hat{b}_{i j}$ have a correlation of 0.8855 with $b_{i j}$ ). The specification of drone parameters are as follows (32):

- Sum of drone tare and battery mass: $10.1 \mathrm{~kg}$

- Total battery capacity: $777 \mathrm{Wh}$ 


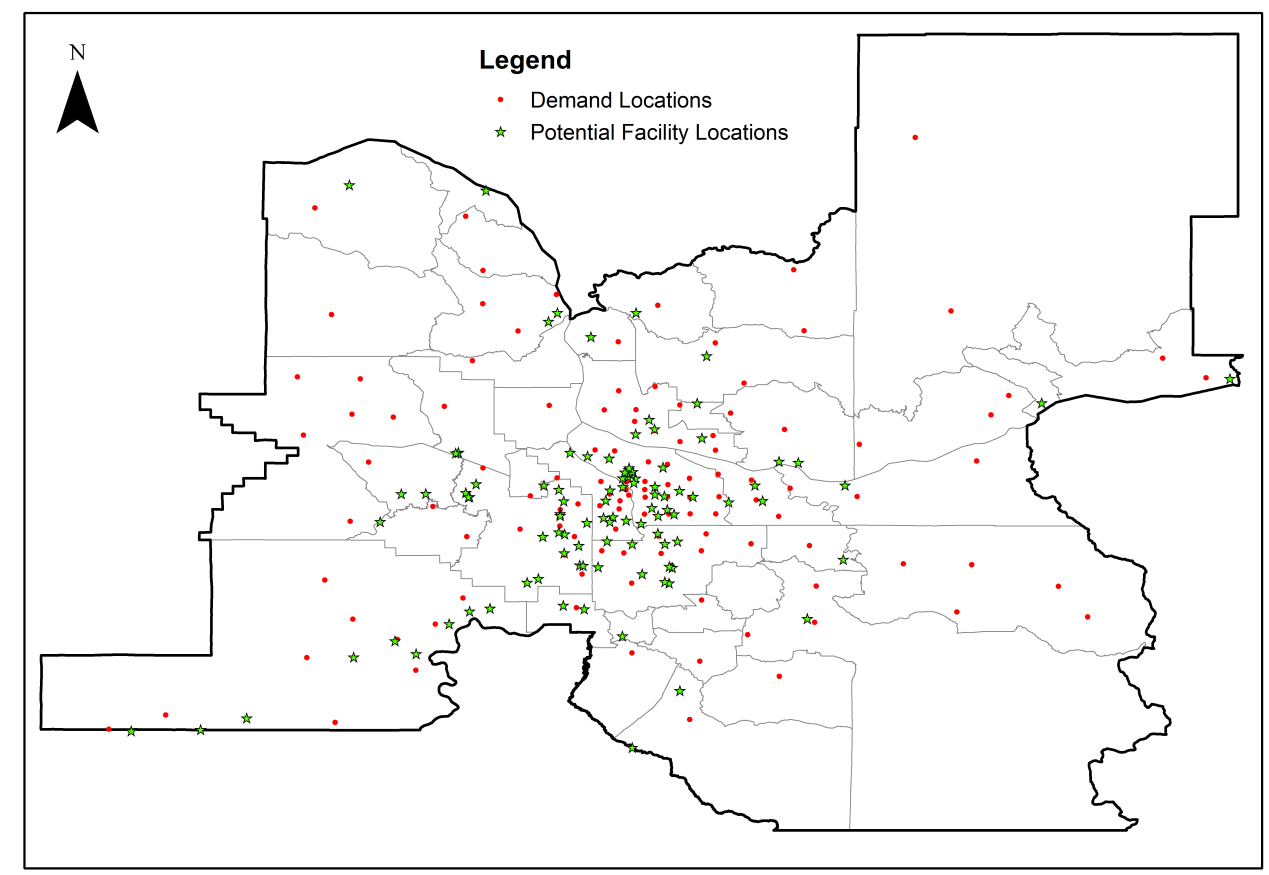

FIGURE 1: Demand locations and potential facility locations in Portland Metro Area (1)

- Payload capacity: $5 \mathrm{~kg}$

- Lift-to-drag ratio: 3.5

- Total power transfer efficiency: 0.66

- Battery Safety Buffer: 20\% of total battery capacity (Maximum usable battery availability $(B)=$ Total battery - Battery Safety Buffer $=621 \mathrm{Wh})$

- Maximum reduction in initial battery availability $\left(f_{k}\right): 25 \%$ of total battery capacity $=$ $194 \mathrm{Wh}$

- Penalty associated with maximum reduction in initial battery availability $\left(F_{k}\right): 2.5 \mathrm{~kg}$

- Maximum number of trips per drone that can assume worst case battery consumption $\left(\Gamma_{j k}\right): 1$

Chauhan et al. (I) found that the 1 facility exchange in the third stage of their proposed $3 \mathrm{SH}$ heuristic works best when $p=5,2$ facility exchange works best when $p=10$, and 3 facility exchange works best for $p$ values greater than 10. As R3SH, in this study, is an extension of $3 \mathrm{SH}$, the above-mentioned values of facility exchange are used in the $r$-exchange heuristic stage of the $\mathrm{R} 3 \mathrm{SH}$. That is:

Value of $r$ in the third stage of R3SH $= \begin{cases}1 & ; \text { if } p=5 \\ 2 & \text {;if } p=10 \\ 3 & ; \text { if } p=15,20,25,30\end{cases}$

The computational analyses for the robust formulations are performed on a Windows 10 desktop with Intel i7-7700K processor with CPU specifications of $3.6 \mathrm{GHz}, 4$ cores, 8 logical processors, and $32 \mathrm{~GB}$ of RAM. The computational analysis on the deterministic formulation of Maximum Coverage Facility Location Problem with Drones which does not consider battery initial 
1 capacity or consumption rate uncertainty $(1)$ is performed on a Windows 10 desktop with Intel i7-

28700 processor with CPU specifications of $3.2 \mathrm{GHz}, 4$ cores, 8 logical processors, and $32 \mathrm{~GB}$ of

3 RAM. The deterministic formulation is solved to evaluate the value of considering uncertainty.

4 Computational Efficiency

5 Computational efficiency for solving RMCFLPD would determine the extent of its application in

6 real life. As the model is developed for short term applications, faster convergence is desired for

7 quicker implementation of drone delivery in the region and faster global reoptimization is desired

8 to tackle changes in demand for resources. The RMCFLPD for the Portland Metro case study is

9 solved using two methods:

- Gurobi solver in Python interface. The model is run for a maximum of 3600 seconds, or a solution within the tolerance limit is obtained. Gurobi default parameters are used for the model.

- Robust three-stage heuristic (R3SH). The facility location problem in stage 1 and the nominal knapsack problems in stage 2 are solved using Gurobi. The $r$-exchange heuristic is repeated 100 times.

The first set of computational runs aim to measure the performance of Gurobi solver versus R3SH. The Gurobi runs are performed once for each combination of $p$ (maximum number of opened facilities) and $|K|$ (Maximum number of drones), and its results can be found in Table 1. As random exchange of facilities is involved in $\mathrm{R} 3 \mathrm{SH}, 30$ runs are performed for each $p-|K|$ combination and the minimum, average, and maximum values are reported.

The objective values achieved by $\mathrm{R} 3 \mathrm{SH}$ are $93.2 \%$ of Gurobi objective values on average (minimum is $88.2 \%$ of Gurobi objective value for the case where $p$ is 15 and $|K|$ is 60; maximum is $97.5 \%$ of Gurobi objective value for the case where $p$ is 30 and $|K|$ is 60 ). R3SH outperforms Gurobi in terms of run times, achieving a median reduction of $97.5 \%$. The first solution to RMCFLPD is generated by R3SH when it completes its second stage (the repeated application of robust knapsack problems) for the first time. This is referred to as $\mathrm{S} 2$ in table 1. At S2, R3SH achieves objective value, which is $88.5 \%$ of the Gurobi objective on average, utilizing a maximum of 3.6 seconds. On average, the third stage of R3SH improves the S2 objective value by $5 \%$ (minimum improvement is $0 \%$ for three unique $p$ - $|K|$ combinations; maximum improvement is $10.8 \%$ for the case when $p$ is 25 and $|K|$ is 50) and adds 105 seconds to the computational time. To compare Gurobi and R3SH at equivalent performance, the times taken by Gurobi to achieve R3SH 'S2' and 'Ave' objective values are noted in Table 2. At this equivalent performance, R3SH is computationally faster than Gurobi, achieving a median reduction in computational time of $98.7 \%$ for 'S2' solution, and $44 \%$ for 'Ave' solution. 
TABLE 1: Comparison of Gurobi solver and R3SH

\begin{tabular}{|c|c|c|c|c|c|c|c|c|c|c|c|c|c|}
\hline \multirow[t]{3}{*}{$p$} & \multirow[t]{3}{*}{$|K|$} & \multicolumn{4}{|c|}{ Gurobi } & \multicolumn{8}{|c|}{ R3SH } \\
\hline & & \multirow{2}{*}{$\begin{array}{c}\text { Time to } 1 \mathrm{st} \\
\text { solution }(\mathrm{sec})\end{array}$} & \multirow[t]{2}{*}{ Time (sec) } & \multirow[t]{2}{*}{ Gap (\%) } & \multirow[t]{2}{*}{ Objective (kg) } & \multicolumn{4}{|c|}{ Time (sec) } & \multicolumn{4}{|c|}{ Objective $(\mathrm{kg})$} \\
\hline & & & & & & $\mathrm{S} 2$ & Min & Ave & Max & $\mathrm{S} 2$ & Min & Ave & $\operatorname{Max}$ \\
\hline 5 & 20 & 21 & 3600 & 2.8 & 216.3 & 2.3 & 99.2 & 112.9 & 132.9 & 194.0 & 194.1 & 200.5 & 203.7 \\
\hline 5 & 25 & 36 & 3600 & 4.4 & 246.1 & 2.5 & 112.4 & 127.4 & 152.9 & 220.4 & 221.4 & 226.0 & 230.0 \\
\hline 5 & 30 & 55 & 3600 & 5.9 & 270.3 & 2.7 & 125.6 & 140.7 & 167.3 & 243.1 & 243.3 & 247.0 & 252.8 \\
\hline 5 & 35 & 69 & 3600 & 7.6 & 289.9 & 3.0 & 165.4 & 175.3 & 190.8 & 263.3 & 263.3 & 265.0 & 267.0 \\
\hline 5 & 40 & 106 & 3600 & 11.5 & 304.0 & 3.6 & 190.0 & 210.1 & 227.4 & 281.9 & 281.9 & 282.7 & 283.3 \\
\hline 10 & 20 & 25 & 3600 & 2.1 & 247.9 & 2.6 & 106.6 & 125.6 & 215.2 & 226.9 & 227.9 & 230.5 & 233.2 \\
\hline 10 & 30 & 50 & 3600 & 4.1 & 303.5 & 2.9 & 134.0 & 146.3 & 164.3 & 270.6 & 274.0 & 278.8 & 283.7 \\
\hline 10 & 40 & 148 & 3600 & 4.8 & 351.4 & 3.2 & 138.9 & 169.7 & 187.6 & 301.6 & 312.2 & 319.9 & 325.5 \\
\hline 15 & 30 & 40 & 3600 & 3.8 & 323.7 & 2.7 & 101.1 & 110.5 & 123.0 & 295.4 & 295.4 & 299.4 & 304.3 \\
\hline 15 & 45 & 264 & 3600 & 5.0 & 394.4 & 2.5 & 104.5 & 117.9 & 128.9 & 344.5 & 359.3 & 364.5 & 372.3 \\
\hline 15 & 60 & 504 & 3600 & 6.0 & 448.3 & 2.2 & 92.4 & 106.8 & 120.9 & 382.0 & 395.2 & 405.4 & 419.2 \\
\hline 20 & 20 & 19 & 3600 & 0.5 & 276.4 & 1.8 & 60.0 & 62.8 & 65.8 & 249.7 & 252.9 & 258.8 & 262.1 \\
\hline 20 & 40 & 146 & 3600 & 4.2 & 388.3 & 1.8 & 71.9 & 78.6 & 82.9 & 345.6 & 355.4 & 360.2 & 364.5 \\
\hline 20 & 60 & 340 & 3600 & 4.2 & 461.2 & 1.8 & 72.7 & 83.4 & 88.9 & 395.6 & 416.3 & 425.0 & 431.3 \\
\hline 20 & 80 & 410 & 3600 & 3.2 & 516.5 & 1.8 & 72.9 & 82.6 & 89.0 & 445.6 & 466.3 & 475.0 & 481.3 \\
\hline 25 & 25 & 24 & 3600 & 1.3 & 315.0 & 1.5 & 54.5 & 59.5 & 66.0 & 281.6 & 285.6 & 290.7 & 296.9 \\
\hline 25 & 50 & 193 & 3600 & 3.5 & 438.0 & 1.6 & 73.5 & 78.2 & 84.6 & 381.3 & 409.2 & 417.7 & 422.6 \\
\hline 25 & 75 & 343 & 3600 & 2.2 & 509.5 & 1.6 & 72.9 & 78.5 & 89.1 & 443.8 & 472.0 & 484.1 & 491.1 \\
\hline 25 & 100 & 264 & 3600 & 1.5 & 574.5 & 1.6 & 74.3 & 79.4 & 87.3 & 506.3 & 534.5 & 546.6 & 553.6 \\
\hline 30 & 30 & 30 & 3600 & 2.5 & 350.2 & 1.7 & 57.2 & 59.4 & 64.7 & 313.6 & 318.4 & 323.1 & 326.9 \\
\hline 30 & 60 & 202 & 3600 & 2.0 & 474.2 & 1.7 & 71.5 & 75.3 & 78.2 & 420.1 & 446.2 & 455.8 & 462.2 \\
\hline 30 & 90 & 307 & 3600 & 1.1 & 552.3 & 1.7 & 71.6 & 75.9 & 78.9 & 495.1 & 521.2 & 530.8 & 537.2 \\
\hline
\end{tabular}

S2: After Stage 2 of R3SH 
TABLE 2: Comparison of Gurobi solver and R3SH at equivalent performance

\begin{tabular}{|c|c|c|c|c|c|c|c|}
\hline \multirow{2}{*}{$p$} & \multirow{2}{*}{$|K|$} & \multicolumn{2}{|c|}{ 3SH Ojective $(\mathrm{kg})$} & \multicolumn{2}{|c|}{ 3SH Time (sec) } & \multicolumn{2}{|c|}{$\begin{array}{c}\text { Gurobi } \\
\text { Time }\end{array}$} \\
\hline & & S2 & Ave & $\mathrm{S} 2$ & Ave & $\mathrm{S} 2$ & Ave \\
\hline 5 & 20 & 194 & 200.5 & 2.3 & 112.9 & 34 & 88 \\
\hline 5 & 25 & 220.4 & 226 & 2.5 & 127.4 & 135 & 192 \\
\hline 5 & 30 & 243.1 & 247 & 2.7 & 140.7 & 122 & 184 \\
\hline 5 & 35 & 263.3 & 265 & 3 & 175.3 & 384 & 384 \\
\hline 5 & 40 & 281.9 & 282.7 & 3.6 & 210.1 & 149 & 149 \\
\hline 10 & 20 & 226.9 & 230.5 & 2.6 & 125.6 & 53 & 53 \\
\hline 10 & 30 & 270.6 & 278.8 & 2.9 & 146.3 & 70 & 73 \\
\hline 10 & 40 & 301.6 & 319.9 & 3.2 & 169.7 & 186 & 186 \\
\hline 15 & 30 & 295.4 & 299.4 & 2.7 & 110.5 & 68 & 68 \\
\hline 15 & 45 & 344.5 & 364.5 & 2.5 & 117.9 & 549 & 549 \\
\hline 15 & 60 & 382 & 405.4 & 2.2 & 106.8 & 984 & 984 \\
\hline 20 & 20 & 249.7 & 258.8 & 1.8 & 62.8 & 34 & 35 \\
\hline 20 & 40 & 345.6 & 360.2 & 1.8 & 78.6 & 189 & 198 \\
\hline 20 & 60 & 395.6 & 425 & 1.8 & 83.4 & 340 & 735 \\
\hline 20 & 80 & 445.6 & 475 & 1.8 & 82.6 & 410 & 494 \\
\hline 25 & 25 & 281.6 & 290.7 & 1.5 & 59.5 & 39 & 43 \\
\hline 25 & 50 & 381.3 & 417.7 & 1.6 & 78.2 & 202 & 462 \\
\hline 25 & 75 & 443.8 & 1 & 1.6 & 78.5 & 343 & 753 \\
\hline 25 & 100 & 506.3 & 546.6 & 1.6 & 79.4 & 264 & 595 \\
\hline 30 & 30 & 313.6 & 323.1 & 1.7 & 59.4 & 46 & 57 \\
\hline 30 & 60 & 420.1 & 455.8 & 1.7 & 75.3 & 202 & 235 \\
\hline 30 & 90 & 495.1 & 530.8 & 1.7 & 75.9 & 307 & 443 \\
\hline
\end{tabular}

S2: After Stage 2 of R3SH

Ave: Average solution obtained by R3SH

\section{Value of adding robustness}

2 This section shows the value of adding robustness to the deterministic model, thereby providing a 3 comparison between the robust formulation presented in this paper and the deterministic formula4 tion presented in Chauhan et al. (1). The deterministic formulation is solved exactly using Gurobi 5 with a maximum computational time of $3600 \mathrm{sec}$. The robust formulation is solved using R3SH, 6 as in the previous section.

In order to get a clearer idea of the value of considering robustness and uncertainty, we 8 use Monte-Carlo simulation to generate scenarios. In each scenario, we generate new battery 9 consumption as: $\tilde{b}_{i j} \in \operatorname{Uniform}\left(b_{i j}-\hat{b}_{i j}, b_{i j}+\hat{b}_{i j}\right) \forall i \in I, j \in J$ and new fraction of reduction 10 initial battery availability as: $\tilde{\delta}_{j k} \in \operatorname{Uniform}(0,1) \forall j \in J, k \in K$. The solutions obtained from the 11 robust optimization formulation and deterministic formulation is compared for the new values of $12 \tilde{b}_{i j} \forall i \in I, j \in J$ and $\tilde{\delta}_{j k} \forall j \in J, k \in K$. The key comparison statistics of interest are the percentage 13 of times a drone delivery schematic needs to be recalculated (because of battery capacity constraint 14 violation) and actual demand met. The procedure for conducting the Monte Carlo simulation is 


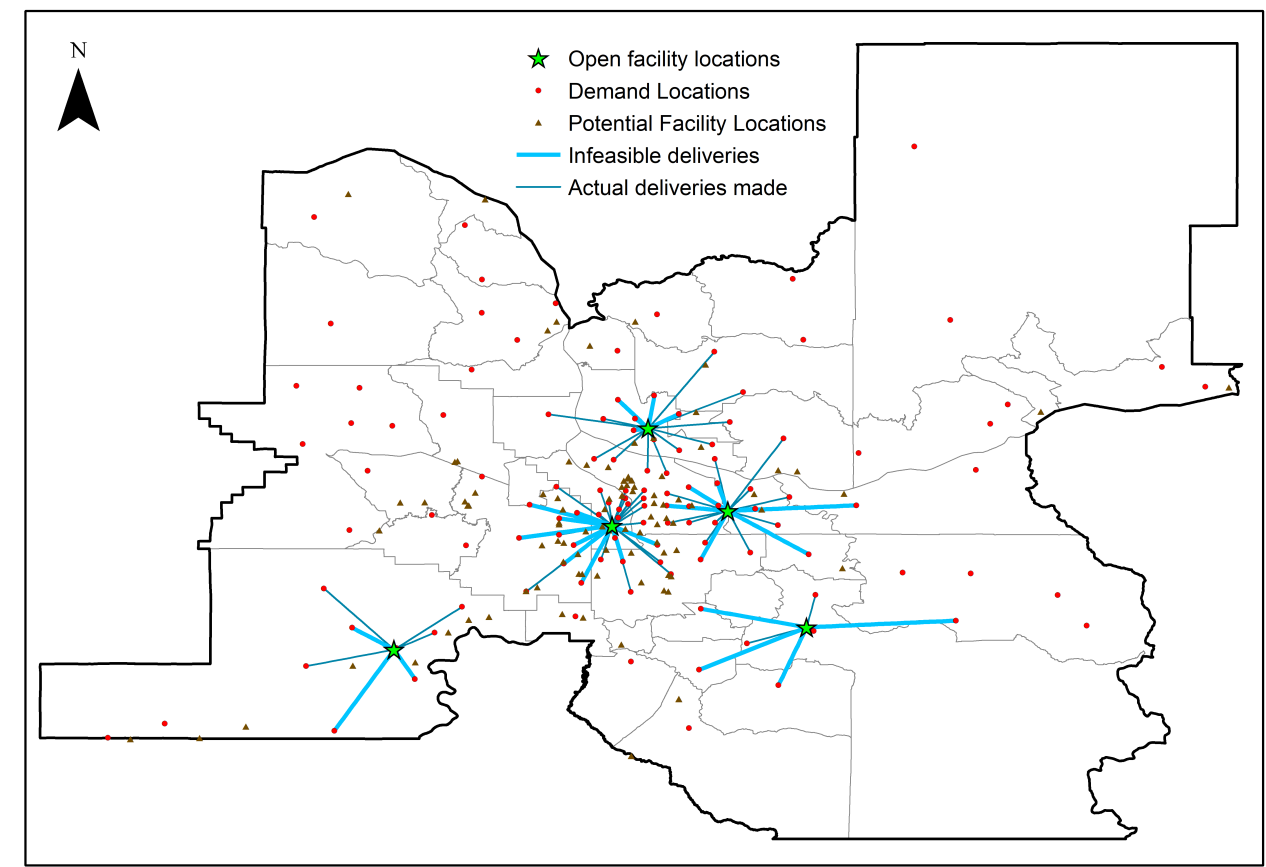

FIGURE 2: Drone delivery scheme using deterministic model with $p=5$ and $|K|=35$ for a simulated value of $\tilde{b}$ and $\tilde{\delta}$

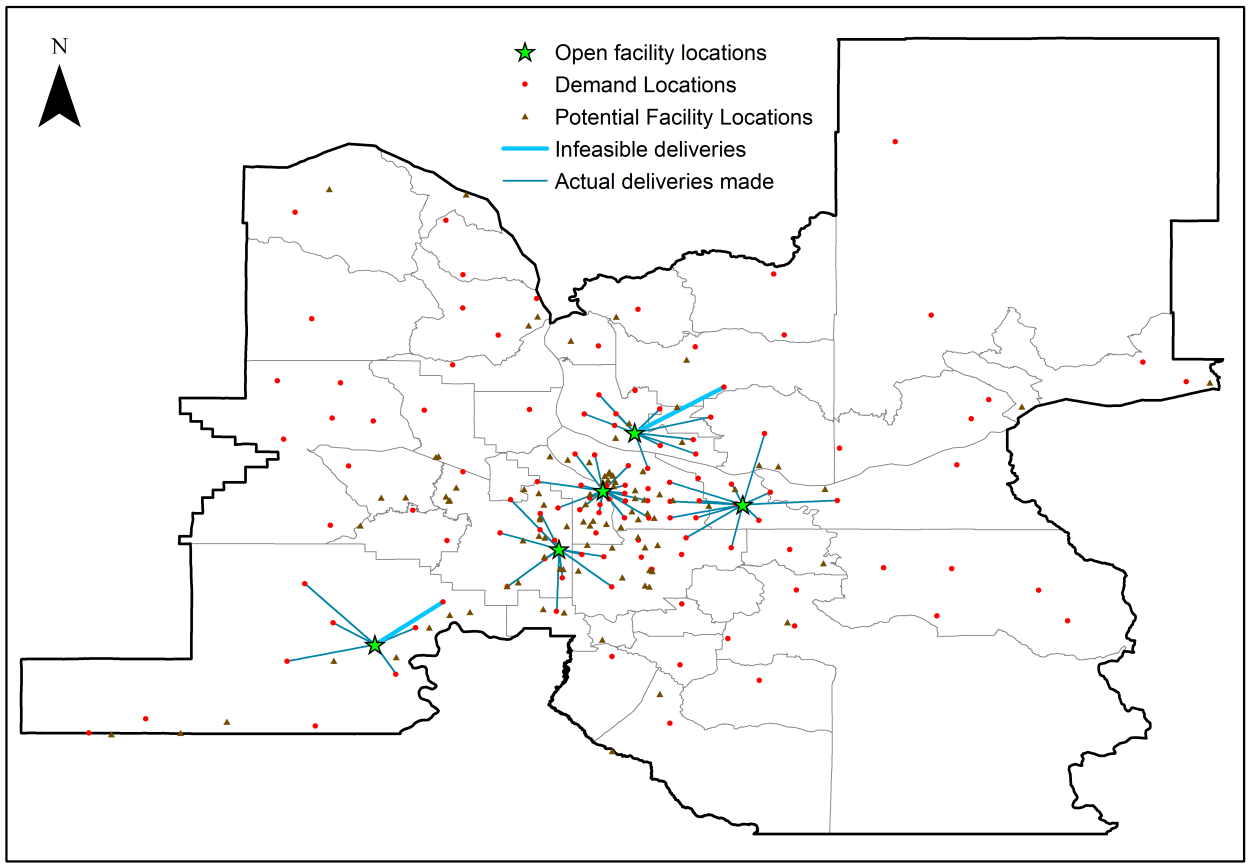

FIGURE 3: Drone delivery scheme using robust model with $p=5$ and $|K|=35$ for a simulated value of $\tilde{b}$ and $\tilde{\delta}$ 
1 detailed in algorithm 1 . The drone delivery schemes obtained using deterministic and the robust

2 model are shown in Figure 2 and Figure 3, respectively. For both the models, the case with

3 parameters $p=5$, and $|K|=35$ is shown using the same simulated values of $\tilde{b}_{i j}$ and $\tilde{\delta}_{j k}$. It can

4 be noted that for the robust solution the number of drone deliveries out of an open facility is

5 lesser as well as the drone trip lengths are smaller compared to the deterministic solution. This

6 is expected as the robust model is solving for a reduced battery capacity and potentially higher

7 battery consumption rate. As a result, there are a very few infeasible deliveries in the robust case,

8 whereas in the deterministic case, almost half of the deliveries proposed were infeasible.

The probability that the delivery schematic for a drone needs to be reevaluated (CVP) is given as the ratio of total_violations and total_constraints, as calculated from algorithm 1. Coverage is calculated as the ratio of total_demand_met to the total demand $(366.5 \mathrm{~kg})$. The probability values along with minimum, average, and maximum values of coverage for the deterministic and robust model is detailed in Table 3 .

The CVP value for the robust formulation is significantly lower than that for the deterministic formulation. The CVP values for the deterministic formulation is particularly high for lower values of $p$. The high values of CVP should result in a greater drop in actual coverage from the coverage reported by the objective function of the model. On average the solution obtained from the deterministic formulation has a CVP of $60.7 \%$ across all $p-K$ combinations tested whereas the corresponding CVP value for the robust solution was 3.6\%. This corresponds to a drop in the actual coverage and erroneous optimistic estimate of the actual coverage when the deterministic model is used. The deterministic model coverage is estimated to be an average of $81.6 \%$ across all $p$ - $K$ combinations. When the deterministic solution is evaluated under battery consumption and battery capacity uncertainty, the actual coverage drops to $64.6 \%$ across all $p-K$ combinations. The robust model provides a more conservative estimate of coverage of $73.8 \%$ across all $p-K$ combinations tested from the optimization model. However, when the robust optimization solution is evaluated using simulation, the actual average coverage is $72.75 \%$ across all $p$ - $K$ combinations. Thus the robust model provides higher and more reliable estimate of actual coverage under uncertainty. The difference between actual coverage of the robust optimization solution and deterministic solution is higher than $5 \%$ when the number of drones is greater than 30 with the difference being as high as $18.4 \%$ for the case when $p$ is 25 and $|K|$ is 75 .

\section{Sensitivity to changes in maximum penalty value}

This section studies the effect of changes in the maximum penalty value $\left(F_{k}\right)$ on the robustness of the solutions. The computational runs for this sensitivity analysis are performed using R3SH and $F_{k}$ values as $2 \mathrm{~kg}, 2.5 \mathrm{~kg}$, and $3 \mathrm{~kg}$. R3SH is run 30 times to provide representative solutions. The robustness of solutions are calculated in the same way as described in the previous section (using algorithm 1). The minimum, average, and maximum values for the probability that the delivery schematic for a drone needs to be reevaluated (CVP) and the coverage is given in Table 4.

The CVP values decrease with increase in the number of available drones for a constant value of $p$ and $F_{k}$, and the CVP values decrease with increase in the $F_{k}$ value for a constant value of $p$ and the number of available drones. As the number of drones increases for a constant value of $p$ and $F_{k}$, the chances of the penalty being accounted in the delivery scheme increases as most locations with high demands are already satisfied by previous drones, and therefore, CVP should decrease. As the value of $F_{k}$ (penalty) increases, the chances of it being favored instead of satisfying the demand points increases, and therefore, CVP should decrease. The increase in $F_{k}$ value from 2 


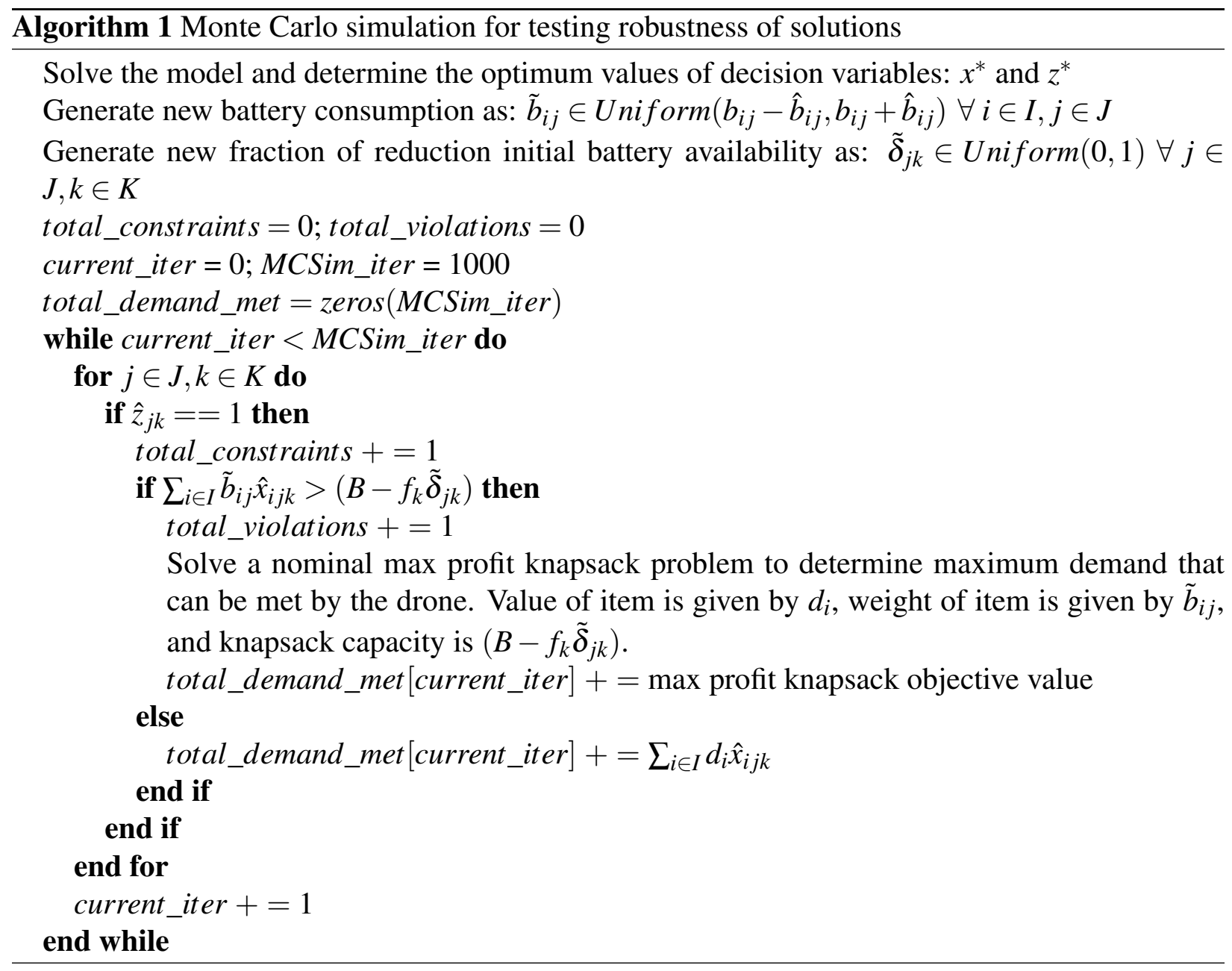

$1 \mathrm{~kg}$ to $2.5 \mathrm{~kg}$ leads to a 4.2 percentage points reduction in CVP on average. An increase in $F_{k}$ value 2 from $2.5 \mathrm{~kg}$ to $3 \mathrm{~kg}$ leads to a further decrease in CVP value by 2 percentage points.

As the number of drones and the number of open facilities increase, the coverage should increase as there are more resources available. As the value of $F_{k}$ increases, there are two counteracting events, viz. the coverage should decrease as the chances of the penalty being favored increases resulting in reduced demand met, and the coverage should increase as the CVP value decreases resulting in reduced infeasible demand assignments. In the current case study, the average actual coverage obtained using $F_{k}$ value of $2.5 \mathrm{~kg}$ is always better than the average actual coverage obtained using $F_{k}$ value of $3 \mathrm{~kg}$ (except for the case when $p=5$ and $|K|=40$ ). The average actual coverage obtained using $F_{k}$ value of $2 \mathrm{~kg}$ is better than that of $F_{k}$ value of $2.5 \mathrm{~kg}$ when a smaller number of drones are employed (less than 50 drones). The $F_{k}$ value of $2.5 \mathrm{~kg}$, therefore, works the best for the case-study as it reasonably hedges against the infeasible demand assignments while providing superior coverage values. 
TABLE 3: Value of adding robustness

\begin{tabular}{|c|c|c|c|c|c|c|c|c|c|c|c|}
\hline \multirow[t]{3}{*}{$p$} & \multirow[t]{3}{*}{$|K|$} & \multicolumn{5}{|c|}{ Deterministic } & \multicolumn{5}{|c|}{ Robust } \\
\hline & & \multirow[t]{2}{*}{ CVP (\%) } & \multirow[t]{2}{*}{$\mathrm{OC}(\%)$} & \multicolumn{3}{|c|}{ Actual Coverage (\%) } & \multirow[t]{2}{*}{ CVP (\%) } & \multirow[t]{2}{*}{$\mathrm{OC}(\%)$} & \multicolumn{3}{|c|}{ Actual Coverage $(\%)$} \\
\hline & & & & Min & Ave & Max & & & Min & Ave & Max \\
\hline 5 & 20 & 84 & 56.3 & 40.8 & 44.8 & 49.9 & 2.8 & 46.6 & 42.4 & 46 & 46.6 \\
\hline 5 & 25 & 77.2 & 61.9 & 42 & 47.8 & 53.4 & 2.4 & 51.7 & 47.1 & 51.1 & 51.7 \\
\hline 5 & 30 & 73.6 & 66.3 & 43 & 49.5 & 55 & 2.4 & 55.2 & 51.5 & 54.4 & 55.2 \\
\hline 5 & 35 & 77.3 & 70.2 & 46.4 & 51.3 & 57.4 & 2.3 & 57.4 & 52.7 & 56.6 & 57.4 \\
\hline 5 & 40 & 76.8 & 72.1 & 44.6 & 50.8 & 58.2 & 2.9 & 58.7 & 54.1 & 57.7 & 58.7 \\
\hline 10 & 20 & 76.2 & 64.3 & 48.8 & 52.7 & 57.6 & 7.4 & 56.3 & 52.3 & 55.1 & 56.3 \\
\hline 10 & 30 & 73.5 & 75.2 & 53 & 58.3 & 64.6 & 6.1 & 65.8 & 60.9 & 64.4 & 65.8 \\
\hline 10 & 40 & 69.7 & 83.5 & 56.8 & 62.9 & 68.4 & 2.8 & 70.9 & 66.1 & 69.8 & 70.9 \\
\hline 15 & 30 & 68 & 80 & 59.3 & 64.6 & 70.5 & 4.3 & 70.8 & 66.1 & 69.9 & 70.8 \\
\hline 15 & 45 & 63.7 & 90.2 & 63.7 & 70.9 & 77.4 & 4.5 & 81.1 & 74.1 & 79.5 & 81.1 \\
\hline 15 & 60 & 53.5 & 92.8 & 63.3 & 71.3 & 78.3 & 1.7 & 84.2 & 78.9 & 83.2 & 84.2 \\
\hline 20 & 20 & 70.1 & 71.4 & 58.7 & 62.2 & 66.5 & 9.5 & 64.7 & 60 & 63.4 & 64.7 \\
\hline 20 & 40 & 58.1 & 90.5 & 68.6 & 74.8 & 81.2 & 3.9 & 82 & 76.7 & 80.7 & 82 \\
\hline 20 & 60 & 57.3 & 93.8 & 63.4 & 71.8 & 79.9 & 1.3 & 87.3 & 82.6 & 86.5 & 87.3 \\
\hline 20 & 80 & 37.5 & 93.8 & 65.3 & 73.7 & 81.4 & 2.3 & 89.9 & 83.2 & 88 & 89.9 \\
\hline 25 & 25 & 57.8 & 79.6 & 65.1 & 69.6 & 74.6 & 8 & 72 & 67.9 & 70.7 & 72 \\
\hline 25 & 50 & 60.6 & 93.8 & 68.1 & 74.8 & 83.1 & 1.8 & 87.7 & 83.8 & 86.7 & 87.7 \\
\hline 25 & 75 & 40.3 & 93.8 & 64.1 & 71.7 & 79.8 & 1.7 & 91.3 & 86.1 & 90.1 & 91.3 \\
\hline 25 & 100 & 25.9 & 93.8 & 67.1 & 75.1 & 82.8 & 0.6 & 90.5 & 87.5 & 89.9 & 90.5 \\
\hline 30 & 30 & 54.2 & 85.7 & 70.3 & 75.1 & 80.1 & 8.4 & 78.9 & 73.9 & 77.1 & 78.9 \\
\hline 30 & 60 & 50.3 & 93.8 & 68.6 & 74.5 & 82.1 & 1.2 & 90 & 86.2 & 89.3 & 90 \\
\hline 30 & 90 & 31.6 & 93.8 & 66.2 & 73.4 & 80.6 & 1.1 & 91.3 & 88 & 90.5 & 91.3 \\
\hline
\end{tabular}

CVP: Probability that the delivery schematic for a drone needs to be reevaluated OC: Coverage calculated using the demand met from objective function of the optimization model 
TABLE 4: Sensitivity to maximum penalty value

\begin{tabular}{|c|c|c|c|c|c|c|c|c|c|c|c|c|c|c|c|c|c|c|c|}
\hline \multirow[t]{2}{*}{$p$} & \multirow[t]{2}{*}{$|K|$} & \multicolumn{6}{|c|}{$F_{k}=2 \mathrm{~kg}$} & \multicolumn{6}{|c|}{$F_{k}=2.5 \mathrm{~kg}$} & \multicolumn{6}{|c|}{$F_{k}=3 \mathrm{~kg}$} \\
\hline & & \multicolumn{3}{|c|}{ CVP (\%) } & \multicolumn{3}{|c|}{ Coverage $(\%)$} & \multicolumn{3}{|c|}{$\operatorname{CVP}(\%)$} & \multicolumn{3}{|c|}{ Coverage (\%) } & \multicolumn{3}{|c|}{ CVP (\%) } & \multicolumn{3}{|c|}{ Coverage (\%) } \\
\hline 5 & 20 & 25.0 & 33.1 & 42.0 & 37.9 & 44.4 & 50.1 & 20.2 & 26.6 & 34.4 & 37.2 & 43.6 & 49.0 & 15.1 & 22.1 & 28.4 & 37.0 & 43.3 & 48.8 \\
\hline 5 & 30 & 16.7 & 21.6 & 29.5 & 43.3 & 50.5 & 56.6 & 15.9 & 19.6 & 25.7 & 44.0 & 50.3 & 56.0 & 13.3 & 16.4 & 19.0 & 43.9 & 50.1 & 55.6 \\
\hline 5 & 35 & 15.6 & 20.1 & 24.2 & 43.5 & 52.3 & 59.4 & 14.8 & 17.0 & 20.0 & 44.8 & 51.5 & 56.5 & 11.8 & 13.4 & 16.2 & 45.8 & 51.4 & 58.1 \\
\hline 5 & 40 & 16.2 & 18.0 & 21.9 & 46.0 & 54.0 & 60.3 & 14.3 & 14.9 & 15.3 & 48.1 & 53.6 & 58.5 & 11.7 & 12.6 & 13.6 & 48.4 & 54.0 & 60.1 \\
\hline 10 & 30 & 17.1 & 22.0 & 28.7 & 52.4 & 59.4 & 65.1 & 9.6 & 17.1 & 21.2 & 52.3 & 58.6 & 63.2 & 5.1 & 11.9 & 15.2 & 51.9 & 58.2 & 61.9 \\
\hline 10 & 40 & 13.8 & 17.0 & 21.4 & 56.6 & 64.1 & 70.6 & 10.1 & 14.1 & 17.5 & 56.7 & 63.6 & 69.4 & 7.6 & 10.6 & 14.1 & 56.8 & 63.2 & 68.2 \\
\hline 15 & 30 & 12.7 & 17.8 & 24.8 & 59.5 & 65.2 & 70.7 & 5.3 & 10.6 & 15.4 & 58.7 & 64.1 & 67.5 & 4.5 & 7.4 & 11.1 & 58.7 & 63.3 & 66.0 \\
\hline 15 & 45 & 9.5 & 14.8 & 18.4 & 64.5 & 72.5 & 78.2 & 6.9 & 10.1 & 13.8 & 64.7 & 72.0 & 77.4 & 4.6 & 7.5 & 10.7 & 65.1 & 71.8 & 77.3 \\
\hline 15 & 60 & 7.8 & 11.4 & 14.2 & 64.3 & 73.4 & 80.6 & 6.1 & 8.4 & 10.1 & 66.1 & 73.5 & 80.6 & 1.2 & 6.2 & 9.0 & 66.7 & 73.5 & 80.2 \\
\hline 20 & 20 & 8.6 & 17.3 & 25.5 & 54.9 & 59.4 & 64.3 & 2.0 & 9.6 & 13.8 & 54.9 & 58.5 & 61.0 & 2.4 & 8.0 & 13.2 & 54.2 & 57.9 & 61.0 \\
\hline 25 & 50 & 5.0 & 7.2 & 10.2 & 75.4 & 82.2 & 86.7 & 2.2 & 3.5 & 5.6 & 75.7 & 82.2 & 85.3 & 1.7 & 3.1 & 6.5 & 75.7 & 81.5 & 84.5 \\
\hline 25 & 75 & 2.9 & 5.0 & 7.4 & 74.9 & 82.4 & 87.9 & 1.4 & 2.6 & 4.5 & 75.9 & 83.3 & 87.7 & 1.2 & 2.5 & 4.3 & 75.3 & 82.3 & 87.4 \\
\hline 25 & 100 & 2.1 & 3.7 & 5.6 & 75.7 & 82.4 & 87.9 & 1.0 & 1.9 & 3.5 & 75.0 & 83.3 & 87.7 & 0.9 & 1.9 & 3.2 & 75.5 & 82.4 & 87.4 \\
\hline 30 & 30 & 5.4 & 9.2 & 12.7 & 65.5 & 70.3 & 74.6 & 1.6 & 4.8 & 6.7 & 65.3 & 69.1 & 71.6 & 0.8 & 4.1 & 8.5 & 65.3 & 69.0 & 72.0 \\
\hline 30 & 60 & 2.9 & 5.0 & 7.8 & 78.2 & 85.2 & 89.2 & 0.8 & 2.5 & 4.4 & 79.2 & 85.7 & 89.2 & 0.8 & 2.4 & 4.3 & 78.7 & 84.7 & 88.5 \\
\hline 30 & 90 & 2.0 & 3.3 & 5.1 & 76.9 & 85.2 & 89.2 & 0.5 & 1.7 & 3.0 & 79.3 & 85.7 & 89.2 & 0.5 & 1.6 & 2.8 & 77.8 & 84.8 & 88.5 \\
\hline
\end{tabular}

CVP: Probability that the delivery schematic for a drone needs to be reevaluated

Note: The numbers have been rounded to nearest tenths for better readability 


\section{CONCLUSIONS}

This paper extends the maximum coverage facility location problem with drones (MCFLPD) proposed by Chauhan et al. (1) by incorporating uncertainty in battery availability and consumption of drones. The uncertainty in initial battery availability is modeled using a penalty-based approach. The higher the penalty, the greater the conservativeness of solution in protecting against gamma robustness principles (34). A mixed-integer linear programming formulation is provided which is solved using Gurobi. As the Gurobi solution time is high, we propose an efficient robust three-stage heuristic (R3SH). The first two stages of the R3SH heuristic obtains a solutions which on an average is within $11 \%$ of the Gurobi solution at 3600 seconds using an average computational time of 2.1 seconds. On an average the R3SH solution are $93 \%$ of the Gurobi solution with a median computational time reduction of $97 \%$.

The robust model provides higher and more reliable estimate of actual coverage under uncertainty. The average difference between actual coverage of the robust optimization solution and deterministic solution is $8.1 \%$ across all $p$ - $K$ (facilities-drones) combination. The difference is higher than $5 \%$ when the number of drones $K$ is greater than 30 with the difference being as high as $18.4 \%$ for the case when the number of facilities is 25 and the number of drones is 75. Incorporating robustness into the deterministic model provides a conservative but reliable coverage estimate, which results in increased actual coverage and reduced number of infeasible drone trips. Also, the probability that the delivery schematic generated by the robust model requires reevaluation on the field is substantially lesser than for the deterministic model, truly highlighting the value considering robustness in decision making.

This work can be extended in multiple directions. One potential extension is the incorporation of one-to-many deliveries where a drone can make multiple deliveries in a single route. The optimization formulation was developed from a coverage maximization perspective which is suitable for disaster relief and other similar applications. Incorporation of sustainability, emissions, and technology (battery replacement) costs can make the model more suitable for urban logistics applications. The methodology proposed here to incorporate uncertainty in battery availability and consumption can also be used in drone-based applications in facility location modeling $(39,40)$ and routing-based applications $(15,23,41)$. A key potential application of drone-based delivery systems is on-demand or real-time dynamic delivery systems. In this case, the number of drones allocated to each facility varies with each time period depending on the dynamic demand. A rolling horizon framework can be used where drone allocations can be made based on current and near-future forecasted demand which can be updated as we receive more information.

\section{ACKNOWLEDGMENTS}

This work was supported by the Data-Supported Transportation Operations and Planning Center, the Center for Advanced Multimodal Mobility Solutions and Education, the Freight Mobility Research Institute, and the National Science Foundation under grants CMMI-1254921: CAREER: Integrated Multiresolution Transportation Network Modeling, CMMI-1562109/1562291: Collaborative Research: Non-Additive Network Routing and Assignment Models, and CMMI1636154: Optimal Control of a Swarm of Unmanned Aerial Vehicles for Traffic Flow Monitoring in Post-disaster Conditions. 


\section{AUTHOR CONTRIBUTIONS}

2 The authors confirm contribution to the paper as follows: study conception and design:

3 D.R. Chauhan, A. Unnikrishnan, M. Figliozzi, S. Boyles; data collection: D.R. Chauhan; analysis 4 and interpretation of results: D.R. Chauhan, A. Unnikrishnan; draft manuscript preparation: D.R.

5 Chauhan, A. Unnikrishnan, M. Figliozzi, S. Boyles. All authors reviewed the results and approved 6 the final version of the manuscript. 


\section{REFERENCES}

2 1. Chauhan, D., A. Unnikrishnan, and M. Figliozzi, Maximum coverage capacitated facility

3 location problem with range constrained drones. Transportation Research Part C: Emerging Technologies, Vol. 99, 2019, pp. 1-18.

2. Karaca, Y., M. Cicek, O. Tatli, A. Sahin, S. Pasli, M. F. Beser, and S. Turedi, The potential use of unmanned aircraft systems (drones) in mountain search and rescue operations. American Journal of Emergency Medicine, Vol. 36, 2018, pp. 585-588.

3. Thiels, C., J. Aho, S. Zietlow, and D. Jenkins, Use of Unmanned Aerial Vehicles for Medical Product Transport. Air Medical Journal, Vol. 34, No. 2, 2015, pp. 104-108.

4. Scott, J. E. and C. H. Scott, Models for Drone Delivery of Medications and Other Healthcare Items. International Journal of Healthcare Information Systems and Informatics (IJHISI), Vol. 13, No. 3, 2018, pp. 20-34.

5. Albornoz, C. and L. F. Giraldo, Trajectory design for efficient crop irrigation with a UAV. In 2017 IEEE 3rd Colombian Conference on Automatic Control (CCAC), IEEE, 2017, pp. $1-6$.

6. Berner, B. and J. Chojnacki, Use of drones in crop protection. In IX International Scientific Symposium "Farm Machinery and Processes Management in Sustainable Agriculture", IEEE, 2017, pp. 46-51.

7. Burema, H. and A. Filin, Aerial farm robot system for crop dusting, planting, fertilizing and other field jobs, 2016, uS Patent 9,382,003.

8. Mack, E., How Delivery Drones Can Help Save The World, 2018, accessed: May 2018.

9. Hassanalian, M. and A. Abdelkefi, Classifications, applications, and design challenges of drones: A review. Progress in Aerospace Sciences, Vol. 99, 2017, pp. 99-131.

10. Yanmaz, E., S. Yahyanejad, B. Rinner, H. Hellwagner, and C. Bettstetter, Drone networks: Communications, coordination, and sensing. Ad Hoc Networks, Vol. 68, 2018, pp. 1-15.

11. Fehrenbacher, K., A New Lithium-Metal Battery Takes Flight in Drones, 2018, accessed: May 2018.

12. FAA, FAA Begins Drone Airspace Authorization Expansion, 2018, accessed: May 2018.

13. Murray, C. C. and A. G. Chu, The flying sidekick traveling salesman problem: Optimization of drone-assisted parcel delivery. Transportation Research Part C: Emerging Technologies, Vol. 54, 2015, pp. 86-109.

14. Ponza, A., Optimization of drone-assisted parcel delivery. Master's thesis, University of Padova, 2016.

15. Agatz, N., P. Bouman, and M. Schmidt, Optimization approaches for the traveling salesman problem with drone. Transportation Science, 2018.

16. Carlsson, J. G. and S. Song, Coordinated logistics with a truck and a drone. Management Science, 2017.

17. Yurek, E. E. and H. C. Ozmutlu, A decomposition-based iterative optimization algorithm for traveling salesman problem with drone. Transportation Research Part C: Emerging Technologies, Vol. 91, 2018, pp. 249-262.

18. Ha, Q. M., Y. Deville, Q. D. Pham, and M. H. Hà, On the min-cost traveling salesman problem with drone. Transportation Research Part C: Emerging Technologies, Vol. 86, 2018, pp. 597-621.

19. Wang, X., S. Poikonen, and B. Golden, The vehicle routing problem with drones: Several worst-case results. Optimization Letters, Vol. 11, No. 4, 2017, pp. 679-697. 
1 20. Poikonen, S., X. Wang, and B. Golden, The vehicle routing problem with drones: Ex2 tended models and connections. Networks, Vol. 70, No. 1, 2017, pp. 34-43.

3 21. Daknama, R. and E. Kraus, Vehicle Routing with Drones. eprint arXiv:1705.06431, 2017.

4 22. Dayarian, I., M. Savelsbergh, and J.-P. Clarke, Same-Day Delivery with Drone Resupply.

23. Dorling, K., J. Heinrichs, G. G. Messier, and S. Magierowski, Vehicle routing problems for drone delivery. IEEE Transactions on Systems, Man, and Cybernetics: Systems, Vol. 47, No. 1, 2017, pp. 70-85.

24. Choi, Y. and P. M. Schonfeld, Optimization of Multi-package Drone Deliveries Considering Battery Capacity. In 96th Annual Meeting of the Transportation Research Board, Washington, DC (Paper No. 17-05769), 2017.

25. Chowdhury, S., A. Emelogu, M. Marufuzzaman, S. G. Nurre, and L. Bian, Drones for disaster response and relief operations: a continuous approximation model. International Journal of Production Economics, Vol. 188, 2017, pp. 167-184.

26. Golabi, M., S. M. Shavarani, and G. Izbirak, An edge-based stochastic facility location problem in UAV-supported humanitarian relief logistics: a case study of Tehran earthquake. Natural Hazards, Vol. 87, No. 3, 2017, pp. 1545-1565.

27. Pulver, A. and R. Wei, Optimizing the spatial location of medical drones. Applied Geography, Vol. 90, 2018, pp. 9-16.

28. Kim, S. J., G. J. Lim, J. Cho, and M. J. Côté, Drone-aided healthcare services for patients with chronic diseases in rural areas. Journal of Intelligent \& Robotic Systems, Vol. 88, No. 1, 2017, pp. 163-180.

29. Kim, S. J., G. J. Lim, and J. Cho, Drone flight scheduling under uncertainty on battery duration and air temperature. Computers \& Industrial Engineering, Vol. 117, 2018, pp. 291-302.

30. Kim, D., K. Lee, and I. Moon, Stochastic facility location model for drones considering uncertain flight distance. Annals of Operations Research, 2018, pp. 1-20.

31. Goodchild, A. and J. Toy, Delivery by drone: An evaluation of unmanned aerial vehicle technology in reducing CO2 emissions in the delivery service industry. Transportation Research Part D: Transport and Environment, 2017.

32. Figliozzi, M., Lifecycle modeling and assessment of unmanned aerial vehicles (Drones) CO2e emissions. Transportation Research Part D, Vol. 57, 2017, pp. 251-261.

33. Otto, A., N. Agatz, J. Campbell, B. Golden, and E. Pesch, Optimization approaches for civil applications of unmanned aerial vehicles (UAVs) or aerial drones: A survey. Networks, 2018.

34. Bertsimas, D. and M. Sim, The price of robustness. Operations research, Vol. 52, No. 1, 2004, pp. 35-53.

35. Ben-Tal, A. and A. Nemirovski, Robust solutions of uncertain linear programs. Operations research letters, Vol. 25, No. 1, 1999, pp. 1-13.

36. Lee, C., K. Lee, K. Park, and S. Park, Branch-and-price-and-cut approach to the robust network design problem without flow bifurcations. Operations Research, Vol. 60, No. 3, 2012, pp. 604-610.

37. Bertsimas, D. and M. Sim, Robust discrete optimization and network flows. Mathematical programming, Vol. 98, No. 1-3, 2003, pp. 49-71. 
1 38. Pirkul, H. and D. Schilling, The capacitated maximal covering location problem with 2 backup service. Annals of Operations Research, Vol. 18, No. 1, 1989, pp. 141-154.

3 39. Pinto, R., M. Zambetti, A. Lagorio, and F. Pirola, A network design model for a meal de-

6 40. Lynskey, J., K. Thar, T. Z. Oo, and C. S. Hong, Facility location problem approach for 7

8 41. Zhu, T., S. D. Boyles, and A. Unnikrishnan, Electric Vehicle Travelling Saleman Problem wih Drones. In 99th Annual Meeting of the Transportation Research Board, 2020. 